\title{
Understanding the influence of RESET current due to the active region of phase change memory
}

\author{
Yuchan Wang ${ }^{1 \mathrm{a})}$, Yueqing Wang ${ }^{2}$, Xiaogang $\mathrm{Chen}^{3}$, \\ and Xiaoyun $\mathbf{L i}^{3}$ \\ ${ }^{1}$ School of Optoelectronic Engineering, Chongqing University of Posts and \\ Telecommunications, Chongqing, 400065, China \\ ${ }^{2}$ Shanghai Fudan Microelectronics Group Co., Ltd., Shanghai, 200433, China \\ ${ }^{3}$ State Key Laboratory of Functional Materials for Informatics and Nanotechnology \\ Laboratory, Shanghai Institute of Micro-system and Information Technology, \\ Chinese Academy of Sciences, Shanghai 200050, China
}

a)yuchanwang87@163.com

\begin{abstract}
The RESET current of T-shaped phase change memory (PCM) cells based on $\mathrm{Ge}_{2} \mathrm{Sb}_{2} \mathrm{Te}_{5}$ (GST) with $35 \mathrm{~nm}$ heating electrodes has been studied to understand the influences of RESET current due to the active region via Transmission Electron Microscope (TEM) and testing. The results have been presented and analyzed. Based on the TEM images, it is found that the grains inside and outside the active region have different structures for the operated cells. And the RESET current can be effectively reduced by obtaining larger active region with the grains of Face Centered Cubic (FCC), confirmed by the testing results.
\end{abstract}

Keywords: phase change memory (PCM), RESET, active region

Classification: Electron devices, circuits and modules

\section{References}

[1] S. R. Ovshinsky: "Reversible electrical switching phenomena in disordered structures,” Phys. Rev. Lett. 21 (1968) 1450 (DOI: 10.1103/PhysRevLett.21. 1450).

[2] M. Wuttig and N. Yamada: "Phase-change materials for rewriteable data storage," Nat. Mater. 6 (2007) 824 (DOI: 10.1038/nmat2009).

[3] R. Bez: "Chalcogenide PCM: A memory technology for next decade," IEDM (2010) 1 (DOI: 10.1109/IEDM.2009.5424415).

[4] A. V. Kolobov, et al:: "Understanding the phase-change mechanism of rewritable optical media," Nat. Mater. 3 (2004) 703 (DOI: 10.1038/nmat1215).

[5] H. Chung, et al.: "A $58 \mathrm{~nm} 1.8 \mathrm{~V} 1 \mathrm{~Gb}$ PRAM with $6.4 \mathrm{MB} / \mathrm{s}$ program BW," ISSCC Dig. Tech. Papers (2011) 500 (DOI: 10.1109/ISSCC.2011.5746415).

[6] K. J. Lee, et al.: "A $90 \mathrm{~nm} 1.8 \mathrm{~V} 512 \mathrm{Mb}$ diode-switch PRAM with $266 \mathrm{MB} / \mathrm{s}$ read throughput," ISSCC Dig. Tech. Papers (2007) 472 (DOI: 10.1109/ISSCC. 2007.373499). in Ti-Sb-Te,” Nat. Commun. 5 (2014) 4086 (DOI: 10.1038/ncomms5086). 
[8] Y. Wang, et al:: "Understanding the early cycling evolution behaviors for phase change memory application,” J. Appl. Phys. 116 (2014) 204503 (DOI: 10. 1063/1.4902851).

[9] Y. Wang, et al:: "RESET distribution improvement of phase change memory: The impact of pre-programming," IEEE Electron Device Lett. 35 (2014) 536 (DOI: 10.1109/LED.2014.2308909).

[10] Y. Wang, et al:: "Reduction of reset current in phase change memory by preprogramming," ECS J. Solid State Sci. Technol. 5 (2016) Q13 (DOI: 10.1149/ 2.0161602jss).

\section{Introduction}

Phase change memory (PCM) is a non-volatile solid state memory, and it is now considered as one of the most promising candidates for future memories because of the high read/write speed, good scalability, high retention and robust cycleendurance $[1,2,3,4]$. PCM operations rely on the rapidly reversible phase transition between amorphous and crystalline states. By applying specific electrical pulses with different amplitudes and widths, the phase change material can switch between the amorphous (RESET, high resistance) and crystalline (SET, low resistance) states. As the large different of electrical resistivity between the lowconducting amorphous and high-conducting crystalline, the state of the memory cell is read out nondestructively by measuring its electrical resistance. PCM utilizes the large resistance ratio to store information.

While phase transition processes are well understood and some PCM chips have been manufactured successfully $[5,6]$, there are still some issues need to be solved. And power consumption is one of the most serious in all the issues. So, how to reduce power consumption has become the most hot research topic. Since the most important factor for the RESET power is the current, it makes the RESET current always in focus $[7,8,9,10]$. There are some factors impact the RESET current, and the active region of the phase change materials is one of the most important factors. To understand the influences of RESET current due to the active region, in this work, the RESET current of T-shaped phase change memory (PCM) cells with $35 \mathrm{~nm}$ heating electrodes has been studied via Transmission Electron Microscope (TEM) and numerical simulation. Based on the TEM images and the two-dimensional finite analysis results, it is found that the RESET current can be effectively reduced by obtaining larger active region with the Face Centered Cubic (FCC) grains, which has been confirmed by the actual test results.

\section{Experimental methods}

T-shape PCM test cell fabricated by $40 \mathrm{~nm}$ CMOS technology is applied, as shown in Fig. 1(a). The $35 \mathrm{~nm}$ tungsten (W) BEC, as a heater, is fabricated on a diameter W plug. The $100 \mathrm{~nm}$ thick $\mathrm{Ge}_{2} \mathrm{Sb}_{2} \mathrm{Te}_{5}$ (GST) film is deposited on the BEC layer by physical vapor deposition (PVD). Between the GST film and the W TEC is the TiN layer as the adhesive layer. Fig. 1(b) shows the experimental setup used for currentvoltage (I-V, SET) and resistance-current (R-I, RESET) measurements. DC test is 
used to the current-voltage scanning and sensing the cell resistance, and AC test is applied to carry out the resistance-current tests. The conversion between AC and DC programming paths is used by the switching box. The RESET current pulses can be generated by a high-speed programmable constant current driver chip.

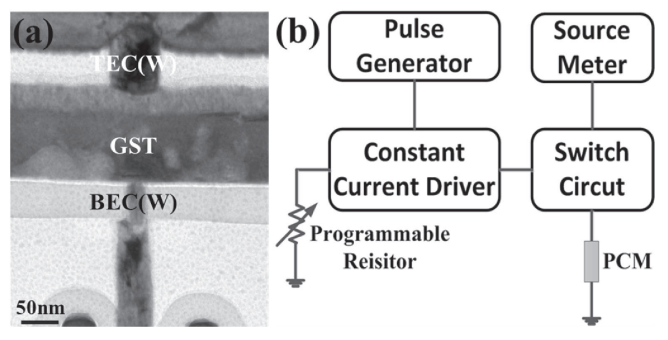

Fig. 1. (a) The TEM image of the cross-sectional structure of the PCM cell and (b) the schematic of experimental setup.

\section{Results and discussion}

In order to determine the initial state of the phase change material, the microscopic study of the cells before any operation is carried out. The cross sections of the cells are prepared by focused ion beam (FIB). The high resolution transmission electron microscope (HRTEM) image of the cell without any operation is presented as shown in Fig. 2(a). To further study the states of the GST, three marked positions (position (1), (2) and (3)) are chosen shown in Fig. 2(a). Fig. 2(b), (c) and (d) show the Fast Fourier Transform (FFT) patterns of the three positions, respectively. Based on the FFT patterns of Fig. 2(b), it can be see that the phase change material above and close to the BEC can be assigned to hexagonal GST, which has been proposed in the Ref. 9. And the FFT patterns of Fig. 2(c) and (d) show that the phase change material far away from the BEC and close to the TEC are all the hexagonal GST. All the results indicate that the PCM never operated is at SET state and all the crystal grains can be assigned to hexagonal GST.

For the operated PCM cells, the state of the phase change material has also been investigated to compare with the initial cells. All the cells have been set at SET state, and the cross sections of the cells are also prepared by focused ion beam (FIB). Fig 3(a) shows the HRTEM image of the cell at SET state. The inset of Fig. 3(a), which is the HRTEM image acquired from the dashed-framed region marked in Fig. 3(a), shows the GST film located above the BEC. And the dashedframed region marked in Fig. 3(a) is almost the central region of the active region. It is found that the film presents a polycrystalline structure in the dashed-framed region, and it finally verifies that these polycrystalline grains are GST with face centered cubic structure (FCC) through the FFT pattern of the dashed-framed region marked in Fig. 3(a) as shown in Fig. 3(b). One marked position has been chosen outside the active region (position (1) as shown in Fig 3(a). Fig. 3(c) shows the FFT pattern of the marked position outside the active region, and it can be found that the grains structure is hexagonal (HEX), which is basically the same with the initial cell shown in Fig. 2(d). For the cells at SET state, it indicates that 
the grains inside the active region are GST with FCC structure, while the grains outside the active region are GST with HEX structure. Fig. 3(d) shows the schematic of the grains state inside and outside the active region of the PCM cell at SET state. This result is consistent with that in Ref. 10, although the BEC material is different for the cells in this work and in Ref. 10.

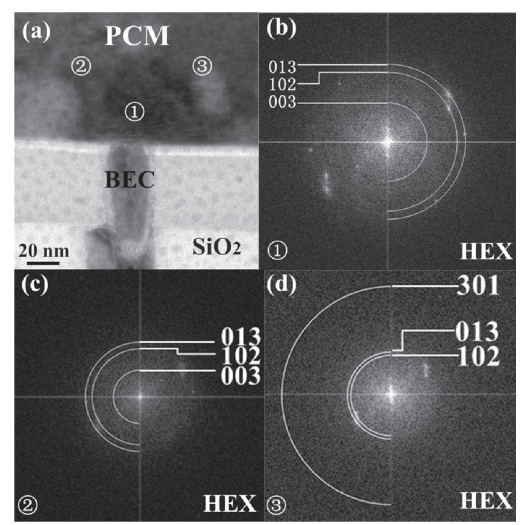

Fig. 2. (a) The TEM bright-filed image showing the cross-section of the PCM cell never operated. The FFT patterns from the positions (1), (2) and (3) marked in (a): (b) shows the pattern of (1), (c) shows the pattern of (2) and (d) shows the pattern of (3).

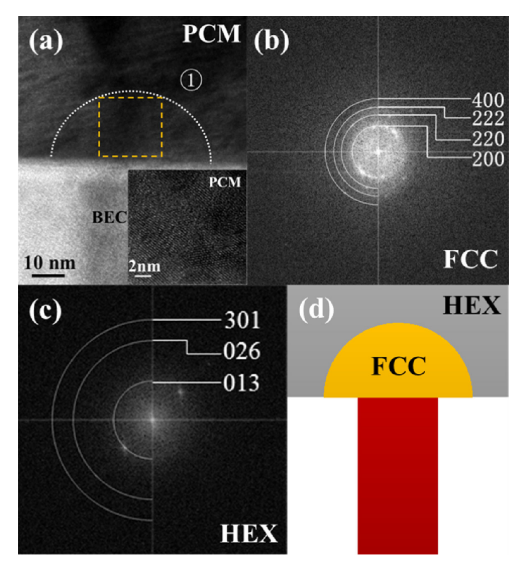

Fig. 3. (a) The TEM image of the PCM cell at SET state after RESETSET operations for many times; (b) The FFT pattern from the square dashed-framed region marked in (a); (c) The FFT pattern from the position (1) marked in (a); (d) Schematic of the PCM cell at steady SET state.

It has been proposed in Ref. 10 that the cell with larger active region (crystalline region with FCC grains) requires less current to achieve the same RESET [10]. In order to further verify the results, two SET-RESET tests have been made to the PCM cells without any operation before. Before the test, the over RESET current value has been first studied. 50 T-shaped cells never operated have been chosen to do the RESET. The cells are set to low resistance state by direct current sweeping (SET), and set to high resistance state by the resistancecurrent (R-I, RESET) measurements. The RESET pulse width is set to $500 \mathrm{~ns}$ with quenching time of $3 \mathrm{~ns}$, and the RESET currents initial value are set to $0.2 \mathrm{~mA}$ with 
a step of $0.1 \mathrm{~mA}$. The read voltage is $0.1 \mathrm{~V}$. The sketch of the RESET pulse applied to the devices is shown in the inset of Fig. 4(a). The maximum current value is set to $1.9 \mathrm{~mA}$, which is the same with that in Ref. 10. The statistics of RESET resistance for each programming current obtained after the RESET are shown in Fig. 4(a) as the red box. Based on the results, it can be found that the median resistance reaches the maximum value as the RESET is about 1.7 or $1.8 \mathrm{~mA}$. When the pre-programming RESET current is beyond 1.7 or $1.8 \mathrm{~mA}$, the median resistance slightly decreases. The results indicate that the over RESET current value is about $1.7 \mathrm{~mA}$. Then the two SET-RESET tests have been carried out. In the test, the PCM cells are divided into two groups. For one group of the cells, the maximum current value of the first RESET is set to $1.6 \mathrm{~mA}$. For the other group of the cells, the maximum current value of the first RESET is set to $1.7 \mathrm{~mA}$. The test results for the first RESET operations are shown in Fig. 4(b). It can be seen that the final RESET resistance for the two groups of the cells are different in the first RESET, which are respectively $1.3 \mathrm{M} \Omega$ and $2.4 \mathrm{M} \Omega$. And the larger RESET current results in the larger final RESET resistance. For the PCM cells, the larger amorphous region will inevitably cause larger amorphous resistance. Therefore, it can indicate that the cells with final RESET resistance $2.4 \mathrm{M} \Omega$ have lager amorphous region, which will result in larger crystalline region with FCC grains in the second SET operation. In the second RESET, the maximum current values are all set to $1.2 \mathrm{~mA}$ for the two groups of the cells. The results of the second RESET operation are also displayed in Fig. 4(b). Comparing the test results of the two groups of PCM cells, it can be found that the RESET current of the second operation have all been reduced, which is consistent with that in Ref. 9. And the cells, which have been applied larger RESET current, need less current to RESET, which means that the cells with lager crystalline region with FCC grains need less current to get the same amorphous resistance.
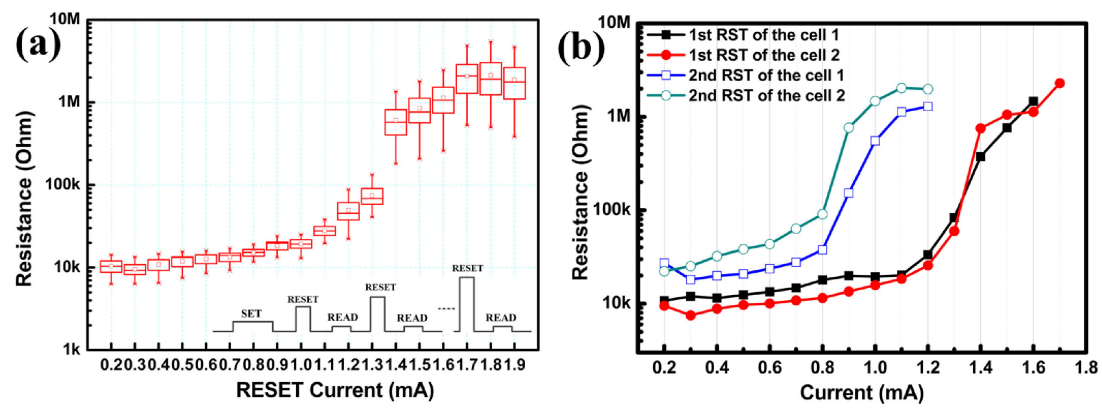

Fig. 4. (a) The statistics of RESET resistance for each programming current obtained after the RESET. (b) R-I performance $s$ of the two PCM cells for the two RESET operations. The inset in (a) shows the sketch of the RESET pulses applied to the cells. 
the TEM images, it is found that the grains inside and outside the active region are GST with FCC structure and GST with HEX structure for the operated cells at SET state. The testing results show that the PCM cells with lager crystalline region with FCC grains need less RESET current to get the same amorphous resistance.

\section{Acknowledgments}

This work was supported by the National Nature Science Foundation of China under Grant No. 61604027, the Basic and Advanced Technology Research Project of Chongqing Municipality under Grant (No. cstc2016jcyjA1923 and No. cstc2016jcyjA0370), the Scientific and Technological Research Foundation of Chongqing Municipal Education Commission under Grant (No. KJ1400411, No. KJ1500404, No. KJ1600439 and No. KJ1709212), the Youth Natural Science Foundation of Chongqing University of Posts and Telecommunications under Grant (No. A2015-50, No. A2015-51, No. A2015-52). 